\title{
Extended focused imaging for digital holograms of macroscopic three-dimensional objects
}

\author{
Conor P. McElhinney, ${ }^{1, \star}$ Bryan M. Hennelly, ${ }^{1}$ and Thomas J. Naughton ${ }^{1,2}$ \\ 'Department of Computer Science, National University of Ireland, Maynooth, County Kildare, Ireland \\ ${ }^{2}$ University of Oulu, RFMedia Laboratory, Oulu Southern Institute, Vierimaantie 5, 84100 Ylivieska, Finland \\ ${ }^{*}$ Corresponding author: conormce@cs.nuim.ie
}

Received 25 October 2007; revised 30 December 2007; accepted 11 January 2008;

posted 24 January 2008 (Doc. ID 88765); published 10 March 2008

\begin{abstract}
When a digital hologram is reconstructed, only points located at the reconstruction distance are in focus. We have developed a novel technique for creating an in-focus image of the macroscopic objects encoded in a digital hologram. This extended focused image is created by combining numerical reconstructions with depth information extracted by using our depth-from-focus algorithm. To our knowledge, this is the first technique that creates extended focused images of digital holograms encoding macroscopic objects. We present results for digital holograms containing low- and high-contrast macroscopic objects. (C) 2008 Optical Society of America
\end{abstract}

OCIS codes: $\quad 090.0090,090.1760,100.6890,100.0100,100.2000$.

\section{Introduction}

Holography is an established technique for recording and reconstructing real-world three-dimensional (3D) objects. Digital holography [1-4] and digital holographic image processing [5-7] have recently become feasible owing to advances in megapixel CCD sensors with high spatial resolution and high dynamic range. In the case of recording, the most commonly used techniques include in-line phase-shifting interferometry (PSI) and off-axis digital holography $[1,3,8]$, which allow the removal of the unwanted intensities and the virtual image that are inherent in holography. A single-exposure on-line setup [9-11] is also possible because of the availability of digital techniques to remove the unwanted terms [1]. In the case of reconstruction, algorithms are applied to the recorded interferogram-holograms in order to view the object under investigation. Numerous techniques exist based on discrete implementations of the Fresnel transform [3]. In the work presented

0003-6935/08/190D71-09\$15.00/0

(C) 2008 Optical Society of America here we employ PSI to capture our in-line digital holograms (DHs), which are in an appropriate form for data transmission and digital image processing.

Digital holographic image processing is a field that has seen increased interest in the recent few years. The large file sizes of DHs have led to research into their compression for the purpose of efficient storage and transmission [12]. The research has focused on compression of the complex field at the hologram plane [5,12-15] and the reconstruction plane [16]. Digital holographic reconstructions also suffer from speckle noise, a noise that is inherent in any optical system using coherent light. The digital nature of DHs allows postprocessing of reconstructions to reduce speckle noise and improve image quality $[17,18]$. Through the use of incoherent object illumination, optical scanning holography produces speckle-free reconstructions [19]. Reconstructions can also be improved through processing of the recorded hologram to remove or suppress error sources such as the twin image [20] and the dc term [1,3]. These terms can also be filtered from the hologram optically $[21,22]$. These properties along with the unique properties of DHs (such as the application of phase-contrast technologies and reconstruction at an arbitrary focal plane) have led to increased 
research in the field of digital holographic microscopy [1,23-26]. The recording of biological data by using digital holographic microscopy has numerous advantages over optical microscopy, such as shape recovery [27,28], real-time tracking of the focal plane [26], and the creation of images where the full sample is in focus [29]. This form of microscopy has also renewed interest in the fields of digital holographic object segmentation [30-32] and recognition [9,33-35].

A disadvantage of holographic reconstructions is the limited depth of field. When a $\mathrm{DH}$ is reconstructed, a distance value $d$ is input as a parameter to the reconstruction algorithm. The depth-of-focus range for a reconstruction using the Fresnel approximation is of the order of a millimeter. For complex 3D scenes, scenes containing multiple objects or containing multiple object features located at different depths, this leads to reconstructions with large blurred regions. We are interested in the creation of an image with an extended depth of field [36], which we are calling an extended focus image (EFI) [29], from sets of digital holographic reconstructions where the full scene is in focus. To do this we must be able to identify when certain object regions are in focus at a given depth. This effectively necessitates the development of a shape extraction technique for macroscopic objects encoded in digital holograms [32,37]. An EFI technique has been previously developed for microscopic objects recorded by using digital holography [29], but the shape extraction technique employed is not applicable to our macroscopic objects primarily because of the corruptive effect of speckle noise, which is not present in DH microscopy.

In this paper we present a method for creating EFIs from sets of digital holographic reconstructions of macroscopic objects. We first create a depth map, using a depth-from-focus (DFF) technique. This technique recovers depth information by calculating a focus measure on blocks of multiple reconstructions of the $\mathrm{DH}$. We then combine the depth map and the reconstructions to create an EFI. We discuss a method for creating an EFI in a short length of time by using a nonoverlapping block approach and a method for creating a qualitatively more accurate EFI by using an overlapping block approach. We also demonstrate EFIs created on both low- and high-contrast scenes and discuss the advantages and disadvantages of the technique. Section. 2 discusses the recording process for PSI DHs and our experimental setup. In Section 3 we introduce focus and focus detection for DHs. The algorithms for calculating a depth map using an overlapping and a nonoverlapping DFF approach are discussed in detail. Section. 4 presents a sequential discussion of our different EFI creation approaches along with their advantages and disadvantages. Section 4 also provides experimental results of EFIs created from DHs encoding real-world 3D objects. In Section $\underline{5}$ some conclusions are drawn about the benefits and limitations of the different EFI creation algorithms.

\section{Phase-Shift Digital Holography}

We record interferograms with an optical system based on a Mach-Zehnder interferometer (see Fig. 1). The interferograms are real-valued images resulting from the interference between an object wave and a reference wave. In our system, a linearly polarized helium-neon $(632.8 \mathrm{~nm})$ laser beam is expanded and collimated and split into object and reference waves. The object wave illuminates an object placed at a distance $d$ that is selected based on the object size in order to avoid aliasing of the CCD [38]. Our CCD camera has $2048 \times 2032$ pixels of size $7.4 \mu \mathrm{m}$ in both dimensions. We designate $U_{0}(x, y)$ as the complex amplitude distribution immediately in front of the 3D object. The reference wave passes through $R P_{1}$ and $R_{2}$, and by selectively rotating the plates we can achieve four phase shift permutations. An interferogram is recorded for every phase shift, and we then use these four interferograms and a four-frame PSI $[4,39]$ algorithm to compute the camera plane complex field $H_{0}(x, y)$, the DH. PSI is a digital holographic technique that calculates in-line holograms free of the twin image and dc term.

We numerically reconstruct a $\mathrm{DH}$ in a plane at any distance $z$ from the camera by using the Fresnel transform $[2,4,33]$. This is achievable because a $\mathrm{DH}$, $H_{0}(x, y)$, contains sufficient amplitude and phase information to reconstruct the complex field $U_{z}(x, y)$. This can be calculated from the Fresnel approximation [40] as

$$
U_{z}(x, y)=\frac{-i}{\lambda z} \exp \left(i \frac{2 \pi}{\lambda} z\right) H_{0}(x, y) \star \exp \left[i \pi \frac{\left(x^{2}+y^{2}\right)}{\lambda z}\right],
$$

where $\lambda$ is the wavelength of the light and $\star$ denotes a convolution. At $z=d$, and ignoring errors in digital propagation due to pixelation and rounding, the discrete reconstruction $U_{Z}(x, y)$ is a close approximation of the physical continuous field $U_{0}(x, y)$. As with conventional holography, a $\mathrm{DH}$ encodes different

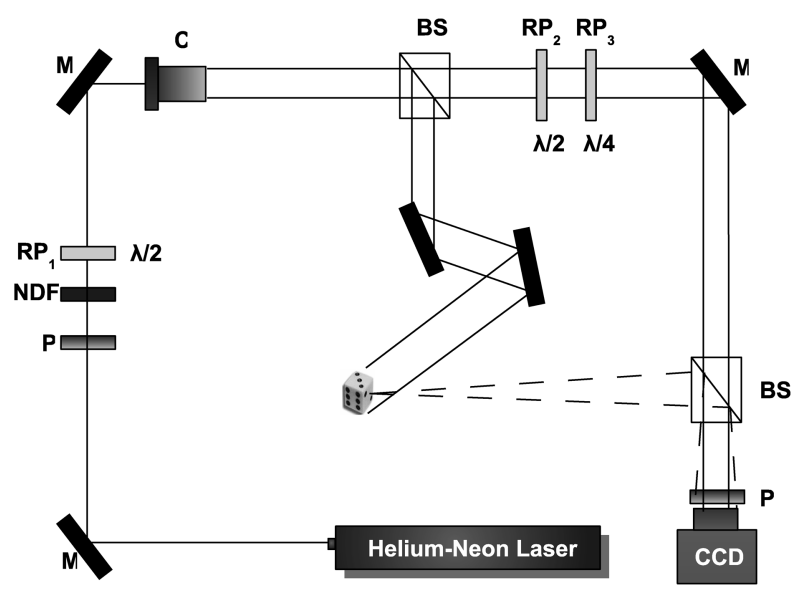

Fig. 1. Experimental setup for PSI: P, polarizer; NDF, neutraldensity filter; C, collimator; $\mathrm{BE}$, beam expander; $\mathrm{BS}$, beam splitter; $\mathrm{RP}$, retardation plate; $\mathrm{M}$, mirror. 
views of a 3D object from a small range of angles $[40,41]$. However, we demonstrate the creation of EFIs using only an individual perspective. In Section 3 we introduce focus and how the relative focus of images can be be calculated.

\section{Focus Detection in Digital Holograms}

Imaging systems generally have a finite depth of field. The recorded image can either be in focus or out of focus. The objects that lie within the depth of field of the imaging system are in focus (appearing sharp), while the objects that lie outside of the depth of field of the system are out of focus (appearing blurred). While the development and application of focus measures in incoherent imaging have been well studied, there exists no definitive criterion for finding the focal plane of a scene or finding the focal distance for a region within a scene. In the field of digital holography, the study of focus measures has not received the same interest. However, focus techniques have been applied to both reconstructions of digitally recorded holograms $[26,29,32,42-44]$ and digitized reconstructions of optically recorded holograms $[37,45,46]$. These employ focus measures such as self-entropy [42], phase changes [26], wavelet analysis [43], gray-level variance [37], and the integrated amplitude modulus [44]. Using these measures, applications such as the detection of the focal plane $[26,43,44]$ in digital holographic microscopy, the measurement of $3 \mathrm{D}$ objects in the digitized reconstructions of physical holograms $[37,47]$, the segmentation of macroscopic objects [32] and the creation of EFIs for microscopic objects [29] have been demonstrated. We now proceed to focus detection and demonstrate how, using a focus measure, we can estimate the depth of pixel blocks in digital holographic reconstructions.

\section{A. Focus Detection}

Focus measures are functions that attempt to determine the relative level of focus of sets of images. The accepted image property maximized by these functions is the high-spatial-frequency energy of the image [48]. Variance is a focus measure calculated on the intensity of an image by using

$$
V\left(I_{z}\right)=\frac{1}{n^{2}} \sum_{i=1}^{n} \sum_{j=1}^{n}\left[I_{z}(i, j)-\overline{I_{z}}\right]^{2},
$$

where $I_{z}$ is an image or image region of size $n \times n$, indexed by pixel locations $i$ and $j$, and where $\overline{I_{z}}$ is the arithmetic mean of $I_{z} . V\left(I_{z}\right)$ is therefore a focus value for the image, or image region, $I_{z}$. Variance has been proved to be a sound focus measure [48]. An example of the application of variance as a focus measure is given in Fig. 2 . We selected one $81 \times 81$ block of pixels on each of the two bolts in the DH. A reconstruction of the $\mathrm{DH}$ with the two blocks highlighted is shown in Fig. 2(a). The variance on the object blocks

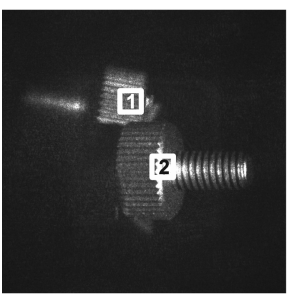

(a)

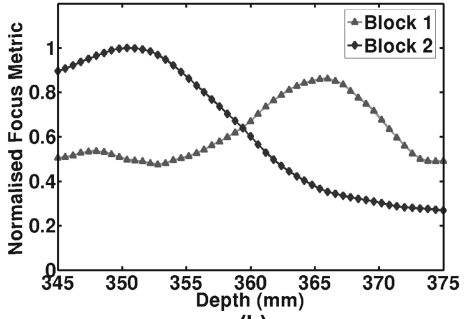

(b)
Fig. 2. Two bolts object DH: (a) numerical reconstruction and (b) variance plot for object blocks 1 and 2 .

is calculated from 151 reconstructions spanning the focal range of the objects in the DH. The focal depth for each object block is selected by choosing the reconstruction depth that returns the highest variance value for that block. The variance plots for the objects blocks are shown in Fig. 2(b). The returned focal depths for the object blocks, obtained by selecting the depth that quantitatively has the maximum focus value, correspond to the depths we obtained by visually selecting the most in-focus depths.

\section{B. Depth from Focus}

DFF is an image processing approach for the estimation of surface shape in a scene using multiple independently focused images. DFF approaches estimate the focal plane of a $\mathrm{DH}$ by maximizing a focus measure, such as Eq. (2), which is applied to the intensity of several 2D reconstructions, where each reconstruction is at a different focal plane. Ma et al. [37] first proposed a DFF algorithm applied to digitized physical holograms for the recovery of $3 \mathrm{D}$ shape information from digitized physical holograms. By calculating variance on nonoverlapping blocks from reconstructions of a $\mathrm{DH}$ at different depths they recovered depth information from a lower-resolution version of the sensed object. We choose to extend this variance-measurement approach in order to classify each $1 \mathrm{D}$ vector $(x, y)$ in the reconstruction volume (each line of pixels parallel to the optical axis) as either belonging to the object or belonging to the background [32]. The decision is taken as follows: if vector $(x, y)$ contains an in-focus pixel from the object at any depth $z$, then $(x, y)$ is an object pixel; otherwise it is a background pixel.

Each reconstruction $I_{z}(k, l)=\left|U_{z}(k, l)\right|^{2}$ is of size $M \times N$ pixels. Our algorithm requires five input parameters: a DH, a block size $n \times n$, a start depth $z_{\min }$, an increment $z_{\text {step }}$, and an end depth $z_{\max }$. The initial reconstruction depth $z$ is set to the starting depth, $z=z_{\min }$. The algorithm involves the following three steps as illustrated in Fig. 3:

Step 1. The input $\mathrm{DH}$ is reconstructed at depth $z$, and a speckle-reduction technique can be applied. Speckle reduction is an optional part of the process, where the speckle-reduction technique to be used is chosen by the user. The output reconstructions intensity is stored in $I_{z}(k, l)$. 


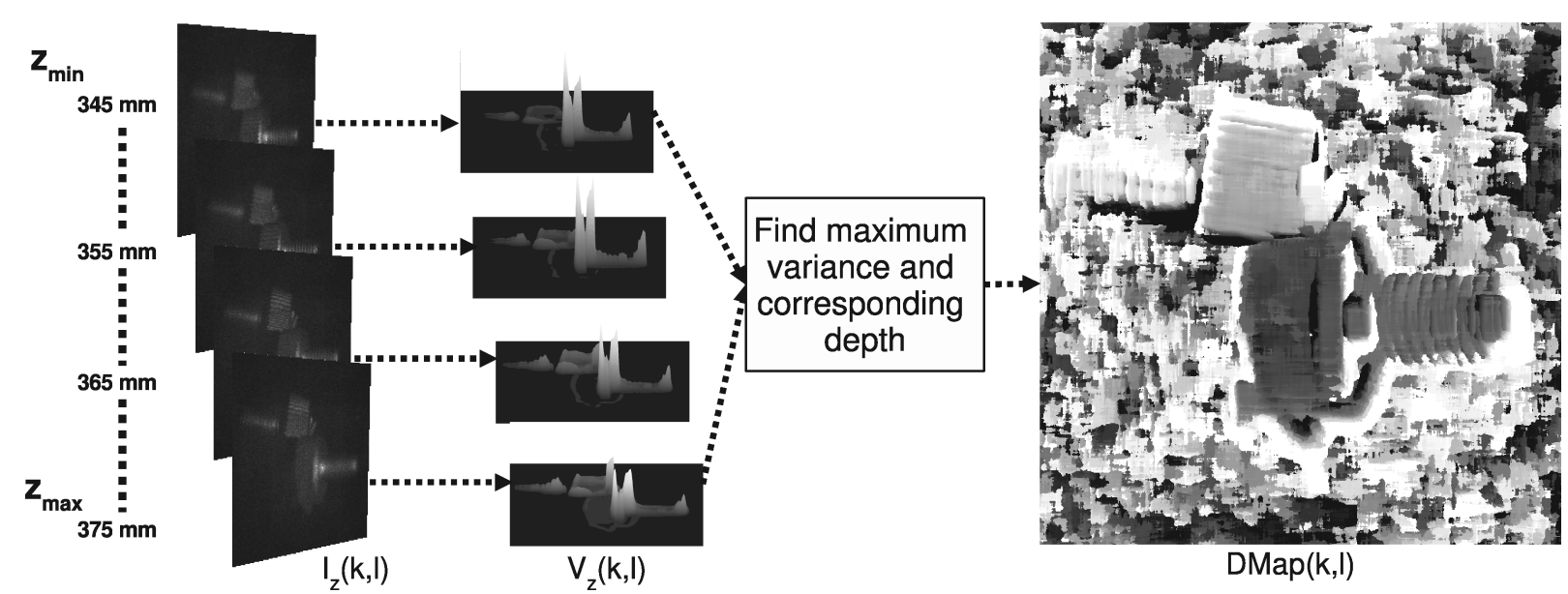

Fig. 3. DFF process: step 1, numerically reconstruct over a range of depths; step 2, block process each reconstruction using variance; step 3, calculate depth map.

Step 2. We then calculate variance for each pixel by calculating variance on $n \times n$ pixel overlapping blocks approximately centered on each pixel, and we address each block as $(k, l)$, where $k \in[0,(M-1)]$, $l \in[0,(N-1)]$. Variance of each overlapping block at each depth $z$ is calculated with the function $V_{z}: \mathbb{R}^{n \times n} \rightarrow \mathbb{R}^{+}$defined by

$$
V_{z}(k, l)=\frac{1}{n^{2}} \sum_{x=k-\left\lfloor\frac{n-1}{2}\right\rfloor}^{k+\left\lceil\frac{n-1}{2}\right\rceil} \sum_{y=l-\left\lfloor\frac{n-1}{2}\right\rfloor}^{l+\left\lceil\frac{n-1}{2}\right\rceil}\left[I_{z}(x, y)-\overline{I_{z}(k, l)}\right]^{2} .
$$

$V$ is therefore a volume storing a $2 \mathrm{D}$ variance image for each depth $z$, and $I_{z}(k, l)$ is defined as

$$
\overline{I_{z}(k, l)}=\frac{1}{n^{2}} \sum_{x=k-\left\lfloor\frac{n-1}{2}\right\rfloor}^{k+\left\lceil\frac{n-1}{2}\right\rceil} \sum_{y=l-\left\lfloor\frac{n-1}{2}\right\rfloor}^{l+\left\lceil\frac{n-1}{2}\right\rceil} I_{z}(x, y) .
$$

For the nonoverlapping algorithm the volume is calculated with

$$
\begin{aligned}
V_{z}^{\mathrm{NO}}(r, s)= & \frac{1}{n^{2}} \sum_{x=r n}^{r n+n-1} \sum_{y=s n}^{s n+n-1}\left[I_{z}(x, y)\right. \\
& \left.-I_{z}\left(r n+\left\lfloor\frac{n-1}{2}\right\rfloor, s n+\left\lfloor\frac{n-1}{2}\right\rfloor\right)\right]
\end{aligned}
$$

where $r \in[0,1, \ldots\lfloor M / n\rfloor-1], s \in[0,1, \ldots\lfloor N / n\rfloor-1]$.

Step 3. The next step is to calculate the depth map. In the overlapping case, for each pixel $(k, l)$ we find the maximum value in $V_{z}(k, l)$. The depth where this maximum occurs, $z$, is then stored in $\operatorname{DMap}(k, l)$. The process is the same for the nonoverlapping case, except we find the maximum value in $V_{z}^{\mathrm{NO}}(r, s)$ and store the depth in $\operatorname{DMap}(r, s)$. This algorithm has been extended to create a maximum variance map that can be employed to segment the object from the background in a $\mathrm{DH}$ reconstruction [32].
We are now in a position to create EFIs by combining $\operatorname{DMap}(k, l)$ with the volume of reconstructions $I_{z}(k, l)$.

\section{Extended Focused Imaging}

In all the experiments in this paper, we use a block size of $81 \times 81$ as input to the DFF algorithm. Larger block sizes have the advantage of estimating the general shape of an object with low error, but the shape of finer object features is lost. Conversely, the smaller block sizes have the advantage of estimating the shape of finer object features but at the cost of high error in the estimate of the general shape of the object. We use the recommended block size of $81 \times 81$ for $\mathrm{DHs}$ containing macroscopic objects [32]. We apply the digital speckle-reduction technique of discrete Fourier filtering developed by Maycock et al. [18] to all our reconstructions; we selected this technique because it has been shown to provide good results for DHs containing macroscopic objects. This speckle-reduction technique is based on applying different nonoverlapping bandpass filters to a complex reconstruction and summing the intensities of the resultant complex images. The depth of focus of the reconstructions used in these experiments ranges from a minimum of 0.4 to a maximum of $0.8 \mathrm{~mm}$ [1]. In this section we demonstrate EFIs with a depth of focus ranging from 6 to $20 \mathrm{~mm}$.

\section{A. Nonoverlapping Case}

By use of the nonoverlapping DFF algorithm, lowresolution depth maps can be created. Through combining a depth map with numerical reconstructions of the $\mathrm{DH}$, we can create EFIs. For each pixel in $\operatorname{DMap}(r, s)$, an $n \times n$ pixel block of intensity values is mapped to the nonoverlapping-case EFI as

$$
\operatorname{EFI}_{\mathrm{NO}}(k, l)=I_{\mathrm{DMap}(r, s)}(k, l),
$$

where $r=\lfloor k / n\rfloor, s=\lfloor l / n\rfloor$ and where the notation $I_{\mathrm{DMap}(r, s)}(k, l)$ denotes the real-valued intensity value 
at lateral coordinates $(k, l)$ and depth $\operatorname{DMap}(r, s)$ in the reconstruction volume, for $k \in\left[0,1, \ldots, M^{\prime}\right], l \in$ $\left[0,1, \ldots, N^{\prime}\right]$ and $M^{\prime}=n\lfloor M / n\rfloor, N^{\prime}=n\lfloor N / n\rfloor$ (for the nonoverlapping case only, the $M^{\prime} \times N^{\prime}$ pixel dimensions of the EFI will be smaller than the original reconstructions if $n$ does not divide $M, N$ ).

In Fig. 4 two numerical reconstructions, from the front focal plane and back focal plane of the $\mathrm{DH}$, are displayed alongside the $\mathrm{EFI}_{\mathrm{NO}}(k, l)$ reconstructions. The objects in this $\mathrm{DH}$ have a depth of focus of approximately $20 \mathrm{~mm}$. We created two $\mathrm{EFI}_{\mathrm{NO}}(k, l)$ reconstructions by using 11 numerical reconstructions and 151 reconstructions, where using a greater number of reconstructions results in a more accurate depth map and therefore a more accurate EFI. To compare these two $\mathrm{EFI}_{\mathrm{NO}}(k, l)$ reconstructions we selected three object regions, which are labeled in Fig. 4: two on the front screw object and one on the back screw object. The numerical reconstructions in Fig. 4 illustrate the limited depth of field of a $\mathrm{DH}$ reconstruction, while the output $\mathrm{EFI}_{\mathrm{NO}}(k, l)$ reconstructions demonstrate how our technique can overcome this limitation to create images where all objects are in focus.
Because of the large size of the DHs, $2048 \times 2048$, the DFF algorithm, and consequently the EFI creation, is computationally intensive. On a $\mathrm{P} 43 \mathrm{GHz}$ personal computer the creation of the $\mathrm{EFI}_{\mathrm{NO}}(k, l)$ using 151 reconstructions takes almost $2.5 \mathrm{~h}$. We investigated the effect of reducing the number of reconstructions on the output EFI for all DHs presented in this paper. By taking the $\mathrm{EFI}_{\mathrm{NO}}(k, l)$ created using a large number of reconstructions (151) as our best EFI, we compared the $\mathrm{EFI}_{\mathrm{NO}}(k, l)$ reconstructions created by using different numbers of reconstructions on two criteria: running time and normalized rms error [49]. Plots of running time and normalized rms error as a function of the number of reconstructions used in $\mathrm{EFI}_{\mathrm{NO}}(k, l)$ creation are shown in Fig 5. The jumps in error value in Fig. 5 occur when our sampling of the reconstruction space does not include the depth for some of the large object regions. This leads to incorrect estimates of depths in object regions, causing blurring in the resulting $\mathrm{EFI}_{\mathrm{NO}}(k, l)$ and an increase in error. For some scenes, it can happen that a lower sampling of depths will include more exact depths at which object regions are located. In a short period of time, less than 10 min, an $\mathrm{EFI}_{\mathrm{NO}}(k, l)$ using only 11 reconstructions
Front Focal Plane
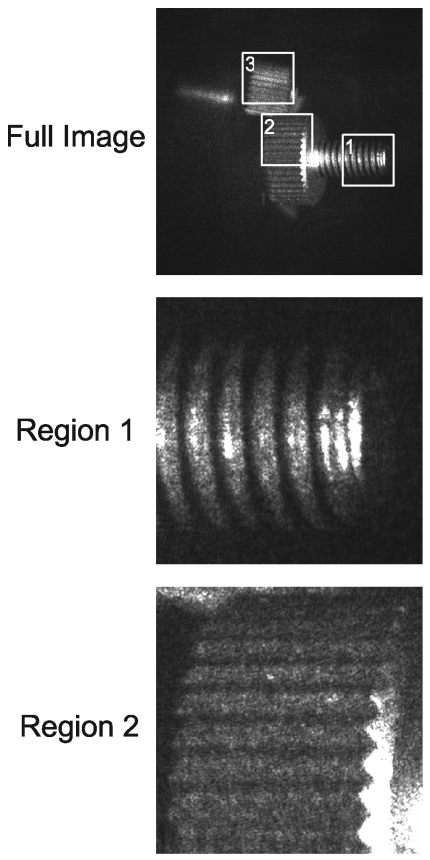

Region 3

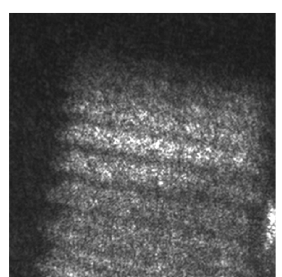

Back Focal Plane
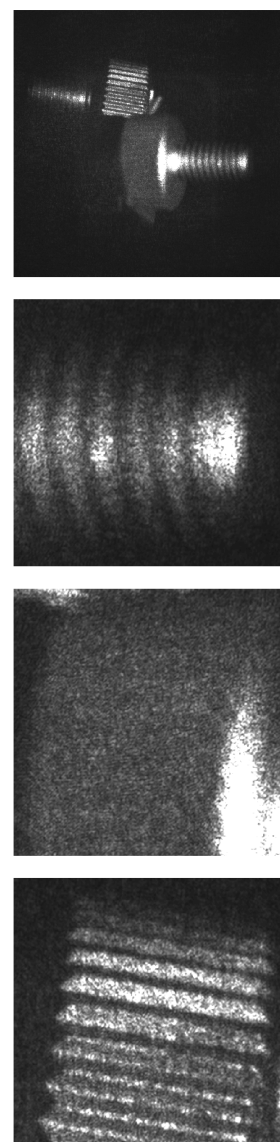
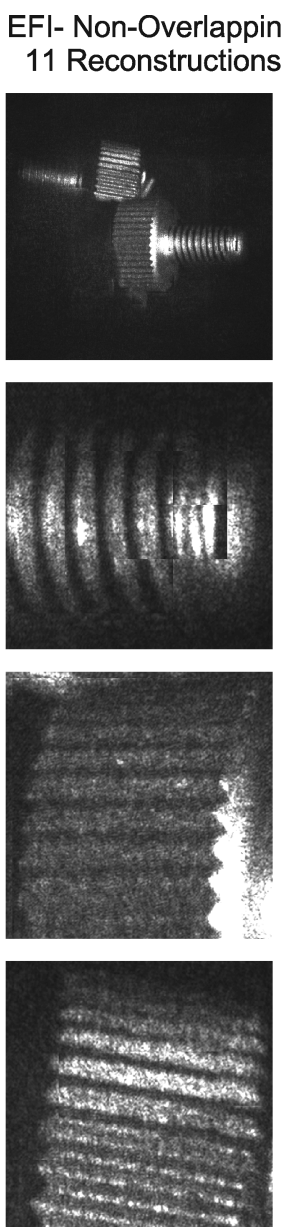
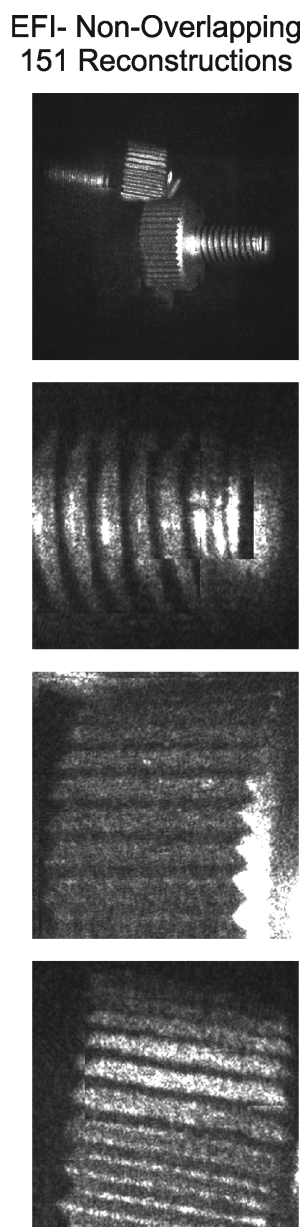

Fig. 4. Two bolts object DH, reconstructions, and the nonoverlapping approach EFIs. 


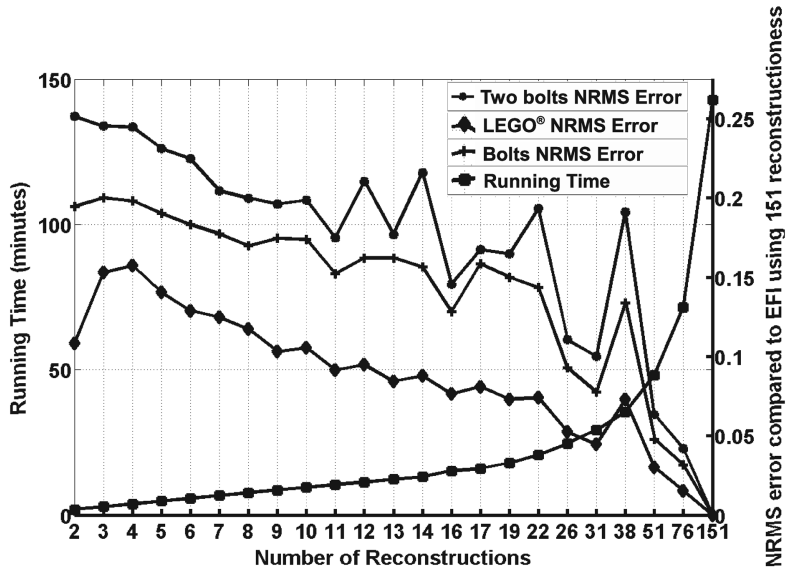

Fig. 5. Accuracy and timing plot for the nonoverlapping approach EFIs created by using increasing numbers of reconstructions.

can be created with an error of $17.5 \%$ when compared with our qualitative best EFI. A visual comparison of the two nonoverlapping EFIs is displayed in Fig. 4. Both of the $\operatorname{EFI}_{\mathrm{NO}}(k, l)$ reconstructions produce images of the scene where both objects are in focus. They also both struggle to select the correct blocks for the tip of the front screw, as shown in the row of region 1 images in Fig. 4. These EFIs can be improved through the use of the overlapping DFF algorithm to create higher-resolution depth maps prior to the calculation of an EFI.

\section{B. Overlapping Approaches}

We have developed two approaches for creating EFIs from depth maps calculated by using the overlapping DFF algorithm. The pointwise approach produces a sharp EFI but does not attempt to compensate for any errors in $\operatorname{DMap}(k, l)$, which can occur for a number of reasons, including poor object illumination or speckle noise. Our neighborhood approach applies smoothing based on the $n \times n$ block size used to create $\operatorname{DMap}(k, l)$. The overlapping algorithm takes, on average, $25 \mathrm{~min}$ to process an individual reconstruction compared with less than $1 \mathrm{~min}$ for the nonoverlapping algorithm. However, The overlapping approach returns a high-resolution $\operatorname{DMap}(k, l)$, allowing a higher-quality EFI.

\section{Pointwise Approach}

To calculate the pointwise $\mathrm{EFI}, \operatorname{EFI}_{\mathrm{P}}(k, l)$, we take the depth for each pixel from $\operatorname{DMap}(k, l)$ and store the intensity value of the corresponding pixel from $I_{z}(k, l)$ for that depth in $\operatorname{EFI}_{\mathrm{P}}(k, l)$. We calculate $\operatorname{EFI}_{\mathrm{N}}(k, l)$ with the following function:

$$
\operatorname{EFI}_{\mathrm{P}}(k, l)=I_{\mathrm{DMap}(k, l)}(k, l),
$$

where $k \in[0,(M-1)], l \in[0,(N-1)]$.

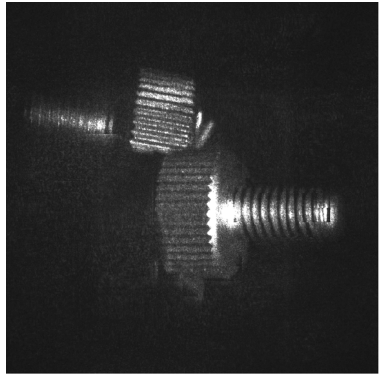

(a)

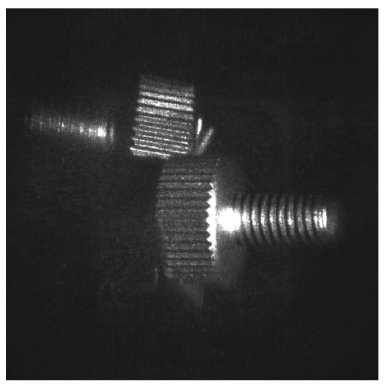

(c)

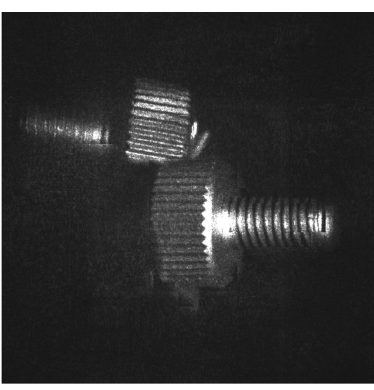

(b)

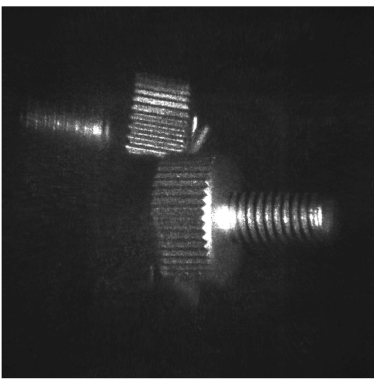

(d)
Fig. 6. Two bolts object EFIs created using the (a) nonoverlapping approach and 11 reconstructions, (b) nonoverlapping approach and 151 reconstructions, (c) overlapping pointwise approach and 151 reconstructions, and (d) overlapping neighborhood approach and 151 reconstructions.

\section{Neighborhood Approach}

We have also developed a second approach for creating EFIs from depth maps created by using the overlapping DFF algorithm. This uses the $n \times n$ block size input to DFF to smooth regions about a neighborhood. For each pixel $(k, l)$ in $\operatorname{DMap}(k, l)$, we take the $n \times n$ pixels, centered on $(k, l)$, from $I_{z}(k, l)$ and store them in the neighborhood EFI, $\operatorname{EFI}_{\mathrm{N}}(k, l)$. This has the effect of reducing the effect of blocks whose depth was incorrectly estimated by summing intensity values for each pixel around a neighborhood. We calculate $\mathrm{EFI}_{\mathrm{N}}(k, l)$ with

$$
\operatorname{EFI}_{\mathrm{N}}(k, l)=\frac{1}{n^{2}} \sum_{r=k-\left\lfloor\frac{n-1}{2}\right\rfloor}^{k+\left\lceil\frac{n-1}{2}\right\rceil} \sum_{s=l-\left\lfloor\frac{n-1}{2}\right\rfloor}^{l+\left\lceil\frac{n-1}{2}\right\rceil} I_{\mathrm{DMap}(r, s)}(k, l),
$$

where $k \in[0,(M-1)], l \in[0,(N-1)]$.

A comparison of the two overlapping approaches, $\operatorname{EFI}_{\mathrm{P}}(k, l)$ and $\operatorname{EFI}_{\mathrm{N}}(k, l)$, and the two $\operatorname{EFI}_{\mathrm{NO}}(k, l)$ reconstructions, detailed in Subsection 4.A, is displayed in Fig. $\underline{6}$. The overlapping approaches produce more accurate EFIs as can be seen by comparing, in particular, object region 1 from the $\mathrm{EFI}_{\mathrm{NO}}(k, l)$, see Fig. $\underline{4}$, with object region 1 from the calculated $\operatorname{EFI}_{\mathrm{P}}(k, l)$ and $\mathrm{EFI}_{\mathrm{N}}(k, l)$, see Fig. 7 . In some cases the depth of object regions may be incorrectly estimated, leading to errors in $\operatorname{EFI}_{\mathrm{P}}(k, l)$. This is apparent in Fig. 7, where there are still some errors in the top of the threads of the screw. 


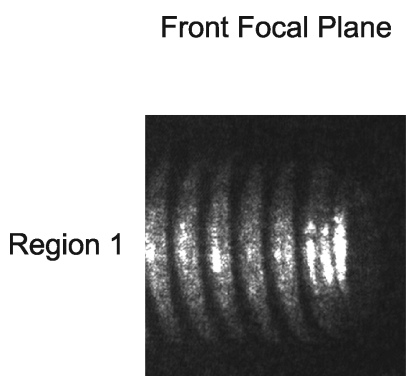

EFI- Pointwise Approach

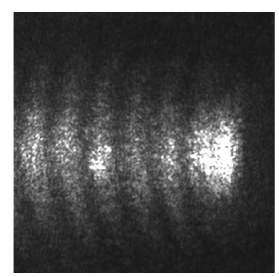

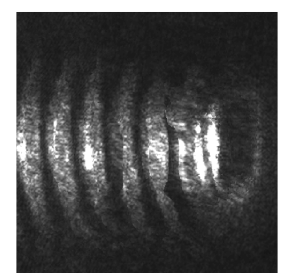

$\mathrm{EFI}-$

Neighborhood

Approach

Fig. 7. Two bolts object DH reconstructions for region 1 and the overlapping approach EFIs.

However, by sacrificing some image sharpness we can reduce these errors by applying the neighborhood approach and calculating $\operatorname{EFI}_{\mathrm{N}}(k, l)$, which has removed the error in the tip of the screw from object region 1 .

To illustrate our techniques' effectiveness on low-contrast objects, we calculated $\operatorname{EFI}_{\mathrm{P}}(k, l)$ and $\operatorname{EFI}_{\mathrm{N}}(k, l)$ for a LEGO block object DH, with a depth of focus of $6 \mathrm{~mm}$. In Fig. 8 a front focal plane and back focal plane reconstruction are shown alongside $\operatorname{EFI}_{\mathrm{P}}(k, l)$ and $\operatorname{EFI}_{\mathrm{N}}(k, l)$. We identified two object regions: the back LEGO block, region 1 , and the front LEGO block, region 2, both with the word "LEGO" inscribed. In these reconstructions the word "LEGO" is legible in only one of the reconstructions, region 2 and region 1 in the front focal and the back focal plane, respectively. However, in $\operatorname{EFI}_{\mathrm{P}}(k, l)$ and $\operatorname{EFI}_{\mathrm{N}}(k, l)$ "LEGO" is legible in both region 1 and region 2. Figure $\underline{9}$ contains a front focal plane recon- struction, a back focal plane reconstruction, and $\operatorname{EFI}_{\mathrm{P}}(k, l)$ for a $\mathrm{DH}$ containing two different types of bolts, where the depth of focus of the objects is $12 \mathrm{~mm}$. Again is it clear that the EFI contains all in-focus regions from both of these images. These EFIs of DHs containing different objects and objects of varying levels of contrast demonstrate the effectiveness of our approach to create focused images out of a volume of $\mathrm{DH}$ reconstructions.

\section{Conclusion}

The field of focus detection and its recent application to digital holographic reconstructions has been discussed in this paper. We have detailed a novel method for creating an image where all objects are in focus, an EFI, out of volumes of digital holographic reconstructions. Using DHs of real-world 3D macroscopic objects, we have experimentally verified our technique. Multiple approaches for creating EFIs

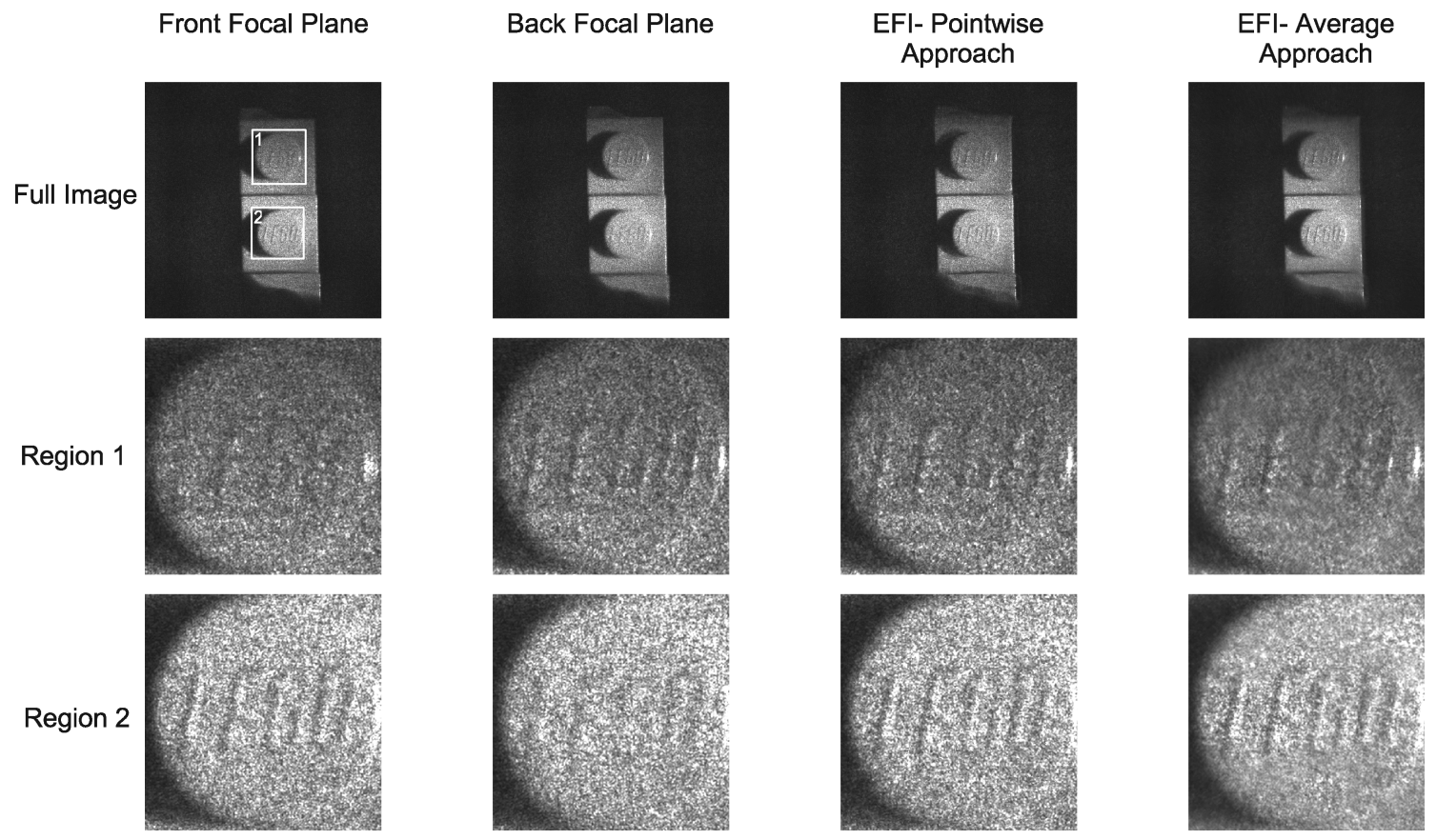

Fig. 8. LEGO block object DH, reconstructions, and the overlapping approach EFIs. 


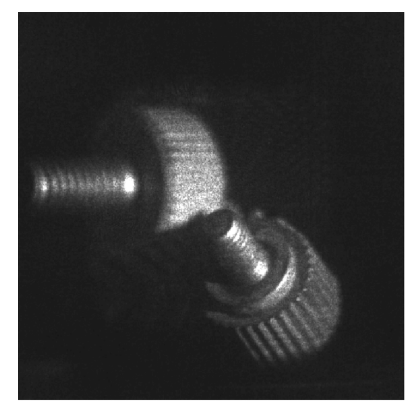

(a)

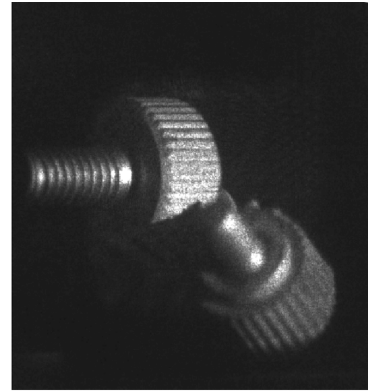

(b)

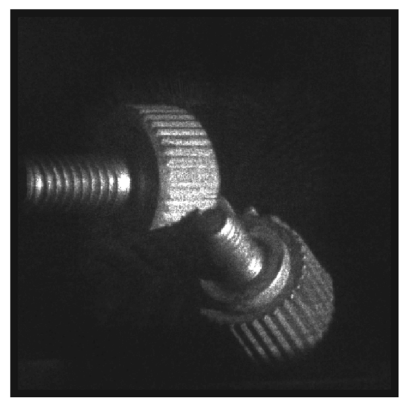

(c)

Fig. 9. Bolts object DH: (a) front focal plane reconstruction, (b) back focal plane reconstruction, (c) $\mathrm{EFI}_{\mathrm{P}}$.

have been described along with their disadvantages and advantages. The nonoverlapping DFF algorithm is relatively fast, but there is a significant loss of resolution. Our overlapping DFF algorithm is computationally expensive but has the advantages of high resolution and, if required, error suppression. We are in the process of parallelizing our DFF algorithm to address the lengthy time required for computing a depth map. Objects can be illuminated with a speckle pattern to increase the accuracy of depth estimation [50], specifically in the case of objects with little or no texture. This EFI creation technique would be suitable for human viewer applications and machine vision applications where the approximate object edges are sufficient (e.g., some object recognition applications). We hope that refinements to the algorithm in the future will make it suitable for general purpose machine vision applications. We intend to investigate the effect of a real physical background behind the objects. We have successfully created EFIs for scenes containing multiple and single objects and containing low- and high-contrast objects and have demonstrated an increase to the depth of focus of our system from 0.8 to $20 \mathrm{~mm}$. To our knowledge, this is the first time that EFIs have been created for DHs containing macroscopic objects.

This publication has emanated from research conducted with the financial support of Science Foundation Ireland, Enterprise Ireland, the Embark Initiative of the Irish Research Council for Science, Engineering, and Technology, and the European Commission through a Marie Curie Fellowship. LEGO is a trademark of the LEGO Group of compa- nies. LEGO did not sponsor, authorize, or endorse this research.

\section{References}

1. T. Kreis, Handbook of Holographic Interferometry (WileyVch, 2005).

2. L. Onural and P. D. Scott, "Digital decoding of in-line holograms," Opt. Eng.. 26, 1124-1132 (1987).

3. U. Schnars and W. Jueptner, Digital Holography: Digital Hologram Recording, Numerical Reconstruction, and Related Techniques (Springer, 2004).

4. I. Yamaguchi and T. Zhang, "Phase-shifting digital holography," Opt. Lett. 22, 1268-1270 (1997).

5. T. J. Naughton, Y. Frauel, B. Javidi, and E. Tajahuerce, "Compression of digital holograms for three-dimensional object reconstruction and recognition," Appl. Opt. 41, 4124-4132 (2002).

6. J. Maycock, C. P. McElhinney, B. H. Hennelly, T. J. Naughton, J. B. McDonald, and B. Javidi, "Reconstruction of partially occluded objects encoded in three-dimensional scenes by using digital holograms." Appl. Opt. 45, 2975-2985 (2006).

7. Y. Frauel, T. J. Naughton, O. Matoba, E. Tajahuerce, and B. Javidi "Three-dimensional imaging and processing using computational holographic imaging," Proc. IEEE 94, 636653 (2006) p. 636.

8. L. P. Yaroslavskii and N. S. Merzlyakov, Methods of Digital Holography, D. Parsons, trans. (Consultants Bureau, 1980).

9. D. Kim and B. Javidi, "Distortion-tolerant 3-D object recognition by using single exposure on-axis digital holography," Opt. Express 12, 5539-5548 (2004).

10. B. Javidi and D. Kim, "Three-dimensional-object recognition by use of single-exposure on-axis digital holography," Opt. Lett. 30, 236-238 (2005).

11. B. Javidi, I. Moon, S. Yeom, and E. Carapezza, "Three-dimensional imaging and recognition of microorganism using singleexposure on-line (SEOL) digital holography," Opt. Express 13, 4492-4506 (2005).

12. T. J. Naughton, J. B. McDonald, and B. Javidi, "Efficient compression of Fresnel fields for Internet transmission of threedimensional images," Appl. Opt. 42, 4758-4764 (2003).

13. I. Yamaguchi, K. Yamamoto, G. A. Mills, and M. Yokota, "Image reconstruction only by phase data in phase-shifting digital holography," Appl. Opt. 45, 975-983 (2006).

14. A. E. Shortt, T. J. Naughton, and B. Javidi, "Compression of digital holograms of three-dimensional objects using wavelets," Opt. Express 14, 2625-2630 (2006).

15. C. P. McElhinney, A. E. Shortt, T. J. Naughton, and B. Javidi, "Blockwise discrete Fourier transform analysis of digital hologram data of three-dimensional objects," Proc. SPIE 5557, 62-69 (2004).

16. E. Darakis and J. J. Soraghan, "Reconstruction domain compression of phase-shifting digital holograms," Appl. Opt. 46, 351-356 (2007).

17. N. Bertaux, Y. Frauel, P. Réfrégier, and B. Javidi, "Speckle removal using a maximum-likelihood technique with isoline gray-level regularization,” J. Opt. Soc. Am. A 21, 2283-2291 (2004).

18. J. Maycock, B. H. Hennelly, J. B. McDonald, T. J. Naughton, Y. Frauel, A. Castro, and B. Javidi, "Reduction of speckle in digital holography by discrete Fourier filtering.” J. Opt. Soc. Am. A 24, 1617-1622 (2007).

19. T.-C. Poon, "Recent progress in optical scanning holography," J. Hologr. Speckle 1, 6-25 (2004).

20. E. Cuche, P. Marquet, and C. Depeursinge, "Spatial filtering for zero-order and twin-image elimination in digital off-axis holography," Appl. Opt. 39, 4070-4075 (2000). 
21. Y. Takari, H. Kawai, and H. Ohzu, "Hybrid holographic microscopy free of conjugate and zero-order images," Appl. Opt. 38, 4990-4996 (1999).

22. T.-C. Poon, T. Kim, G. Indebetouw, B. W. Schilling, M. H. Wu, K. Shinoda, and Y. Suzuki, "Twin-image elimination experiments for three-dimensional images in optical scanning holography," Opt. Lett. 25, 215-217 (2000).

23. T. Zhang and I. Yamaguchi, "Three-dimensional microscopy with phase-shifting digital holography," Opt. Lett. 23, 12211223 (1998).

24. L. Xu, X. Peng, J. Miao, and A. Asundi, "Studies of digital microscopic holography with applications to microstructure testing," Appl. Opt. 40, 5046-5051 (2001).

25. E. Cuche, P. Marquet, and C. Depeursinge, "Simultaneous amplitude-contrast and quantitative phase-contrast microscopy by numerical reconstruction of Fresnel off-axis holograms," Appl. Opt. 38, 6994-7001 (1999).

26. P. Ferraro, G. Coppola, S. Nicola, A. Finizio, and G. Peirattini, "Digital holographic microscope with automatic focus tracking by detecting sample displacement in real time," Opt. Lett. 28, 1257-1259 (2003).

27. G. Pedrini, P. Froning, H. Tiziani, and F. Santoyo, "Shape measurement of microscopic structures using digital holograms," Opt. Commun. 164, 257-268 (1999).

28. C. Mann, L. Yu, C. Lo, and M. Kim, "High-resolution quantitative phase-contrast microscopy by digital holography," Opt. Express 13, 8693-8698 (2005).

29. P. Ferraro, S. Grilli, D. Alfieri, S. D. Nicola, A. Finizio, G. Pierattini, B. Javidi, G. Coppola, and V. Striano, "Extended focused image in microscopy by digital holography," Opt. Express 13, 6738-6749 (2005).

30. M. Gustafsson and M. Sebesta, "Refractometry of microscopic objects with digital holography," Appl. Opt. 43, 4796-4801 (2004).

31. M. Danesh Panah and B. Javidi, "Segmentation of 3D holographic images using bivariate jointly distributed region snake," Opt. Express 14, 5143-5153 (2006).

32. C. P. McElhinney, J. B. McDonald, A. Castro, Y. Frauel, B. Javidi, and T. J. Naughton, "Depth-independent segmentation of three-dimensional objects encoded in single perspectives of digital holograms," Opt. Lett. 32, 1229-1231 (2007).

33. B. Javidi and E. Tajahuerce, "Three-dimensional object recognition by use of digital holography," Opt. Lett. 25, 610-612 (2000).

34. Y. Frauel and B. Javidi, "Neural network for threedimensional object recognition based on digital holography," Opt. Lett. 26, 1478-1480 (2001).
35. B. Javidi, S. Yeom, I. Moon, and M. Daneshpanah, "Real-time automated 3D sensing, detection and recognition of dynamic biological micro-organic events," Opt. Express 14, 3806-3829 (2006).

36. R. J. Pieper and A. Korpel, "Image processing for extended depth of field," Appl. Opt. 22, 1449-1453 (1983).

37. L. Ma, H. Wang, Y. Li, and H. Jin, "Numerical reconstruction of digital holograms for three-dimensional shape measurement," J. Opt. A 6, 396-400 (2004).

38. L. Xu, J. Miao, and A. Asundi, "Properties of digital holography based on in-line configuration," Opt. Eng. 39, 3214-3219 (2000).

39. J. H. Bruning, D. R. Herriott, J. E. Gallagher, D. P. Rosenfeld, A. D. White, and D. J. Brangaccio, "Digital wavefront measuring interferometer for testing optical surfaces and lenses," Appl. Opt. 13, 2693-2703 (1974).

40. J. Goodman, Introduction to Fourier Optics (Roberts and Company, 2005).

41. H. J. Caulfield, Handbook of Optical Holography (Academic, 1979).

42. J. Gillespie and R. King, "The use of self-entropy as a focus measure in digital holography," Pattern Recogn. Lett. 9, 19-25 (1989).

43. M. Liebling and M. Unser, "Autofocus for digital Fresnel holograms by use of a Fresnelet-sparsity criterion," J. Opt. Soc. Am. A 21, 2424-2430 (2004).

44. F. Dubois, C. Schockaert, N. Callens, and C. Yourassowsky, "Focus plane detection criteria in digital holography microscopy," Opt. Express 14, 5895-5908 (2006).

45. E. Malkiel, J. N. Abras and J. Katz, "Automated scanning and measurements of particle distributions within a holographic reconstructed volume," Meas. Sci. Technol. 15, 601-612 (2004).

46. N. Burns and J. Watson, "Data extraction from underwater holograms of marine organisms," in OCEANS 2007-Europe (IEEE, 2007), pp. 1-6.

47. C. P. McElhinney, B. H. Hennelly, J. B. McDonald, and T. J. Naughton are preparing a manuscript to be titled "Multiple object segmentation in macroscopic three-dimensional scenes from a single perspective using digital holography."

48. M. Subbarao, T. Choi, and A. Nikzad," "Focusing techniques," Opt. Eng. 32, 2824-2836 (1993).

49. J. R. Fienup, "Invariant error metrics for image reconstruction," Appl. Opt. 36, 8352-8357 (1997).

50. S. Frey, A. Thelen, S. Hirsch, and P. Hering, "Generation of digital textured surface models from hologram recordings," Appl. Opt. 46, 1986-1993 (2007). 